\title{
F-leap index of some special classes of bridge and chain graphs
}

\begin{abstract}
Mohanad Ali Mohammed $^{\mathrm{a}}{ }^{\circledR}$ |Raad Sehen Haoera $^{\mathrm{a}}$ |Janet Robert $^{\mathrm{b}}{ }^{\circledR}$ |Natarajan Chidambaram $^{\mathrm{b}, *}$
|Narasimhan Devadoss ${ }^{b}$

aDepartment of Mathematics, Open Educational College, Ministry of Education, Al Qadisiya Centre, Iraq

${ }^{b}$ Department of Mathematics, Srinivasa Ramanujan Centre, SASTRA Deemed University, Kumbakonam, Tamil Nadu, India

The 2-degree of a vertex $v$ in a (molecular) graph $G$ is the number of vertices which are at distance two from $v$ in $G$. The F-leap index of a molecular graph $\mathrm{G}$ is the sum of cubes of the 2-degree of every vertex $v$ in $G$. In this research study, we have computed the F-leap index of some special classes of bridges and chain graphs. We also have determined the F-leap index of some chemical structures including polyphenyl chains and spiro chains.
\end{abstract}

\section{*Corresponding Author:}

Natarajan Chidambaram

Email:natarajan_c@maths.sastra.edu

Tel.:+91-9952529575

\section{KEYWORDS}

Leap Zagreb indices; F-leap index; bridge; polyphenyl chain; spiro chain.

\section{Introduction}

A Topological index also known as connectivity index is a type of molecular descriptor that is calculated based on the molecular graph of a chemical compound. It is a numerical parameter of a graph which characterizes its topology and usually graphs invariant. According to [2] topological indices are extensively used as molecular descriptors in building:

- QSAR-Quantitative Structure-Activity Relationship which are extensively used in pharmaceutical and agricultural chemistry for screening compound.

- QSPR-Quantitative Structure-Property Relationship.

- QSTR-Quantitative Structure-Toxicity Relations which is used in predicting toxicity of chemicals.

These have become a powerful tools in contemporary chemical and medicinal research as it is possible to predict the biological activity, specific chemical activity, toxicity and the environmental fate even before its synthesis. An important and oldest topological index introduced by Gutman and Trinajstić [5], and Zagreb index study of structure property and correlation of molecules. They are namely first and second Zagreb indices and defined as follows:

$$
\begin{aligned}
& M_{1}(G)=\sum_{u \in V(G)} \operatorname{deg}(u)^{2}=\sum_{u \in E(G)}[\operatorname{deg}(u)+\operatorname{deg}(v)] \\
& M_{2}(G)=\sum_{u v \in E(G)} \operatorname{deg}(u) \operatorname{deg}(v)
\end{aligned}
$$

Furtula and Gutman [4] defined F-index of graph $\mathrm{G}$ as:

$$
\begin{aligned}
& F(G)=\sum_{u \in V(G)} \operatorname{deg}(u)^{3} \\
& =\sum_{u v \in E(G)}\left[\operatorname{deg}(u)^{2}+\operatorname{deg}(v)^{2}\right]
\end{aligned}
$$

Azari et al. [1] found some interesting results on Zagreb indices of bridges and chain graphs. Infact this is the first seminal paper on topological indices of bridges and chain graph structures .In [8], Nilanjan De computed exact values for the F-index of bridge and chain graphs.

Naji et al. [7] introduced graph invariants based on 2-degree of vertices called Leap Zagreb indices. The 2-degree of a vertex $v$ in $G$ is defined as the number of vertices which are 
at distance two from $\mathrm{v}$ in $\mathrm{G}$ and denoted by $d_{2}(v: G)$ or simply $d_{2}(v)$.

The following are the definitions of leap Zagreb indices given in [7].

First leap Zagreb index:

$$
L M_{1}(G)=\sum_{v \in V(G)} d_{2}(v)^{2}
$$

Second leap Zagreb index:

$$
L M_{2}(G)=\sum_{u v \in E(G)} d_{2}(u) d_{2}(v)
$$

Third leap Zagreb index:

$$
L M_{3}(G)=\sum_{v \in V(G)} \operatorname{deg}(v) d_{2}(v) \text {. }
$$

$\mathrm{X}$. Zhang et al. $[9,10]$ studied the edge version of atom bomb connectivity and geometric arithmetic indices for Cartesian product and join of graphs $[9,10]$. For further research work on these indices refer to [11-17].

In this sequel, V.R. Kulli [6] recently defined another distanced based topological index called F-leap index of a graph $\mathrm{G}$ and defined as $L F(G)=\sum_{v \in V(G)} d_{2}(v)^{3}$. We brief the F-leap index of some well known graphs as follows:

(i) For a path $P_{n}$ on $\mathrm{n}$ vertices, $L F\left(P_{n}\right)=\left\{\begin{array}{l}2, \text { if } n=3 \\ 8 n-28, \text { if } n \geq 4\end{array}\right.$

(ii) For a cycle $C_{n}$ on $\mathrm{n}$ vertices, $L F\left(C_{n}\right)=\left\{\begin{array}{l}0, \text { if } n=3 \\ 8 n, \text { if } n \geq 4\end{array}\right.$

(iii) For a star graph $K_{1, n}$,

$$
L F\left(K_{1, n}\right)=\left\{\begin{array}{l}
0, \text { if } n=1 \\
2, \text { if } n=2 \\
n(n-1)^{3}, \text { if } n \geq 3 .
\end{array}\right.
$$

(iv) It is easy to observe that the $L F\left(K_{n}\right)=0$ as the 2-degree is zero for every vertex in the complete graph $K_{n}$.

(v) For a complete bipartitie graph $K_{r, s}$ with $r \leq s, L F\left(K_{r, s}\right)=r(r-1)^{3}+s(s-1)^{3}$. (vi) For a wheel graph $W_{1, n}$,

$L F\left(W_{1, n}\right)=\left\{\begin{array}{l}0, \text { if } n=3 \\ n(n-3)^{3}, \text { if } n \geq 4 .\end{array}\right.$

\section{Results and discussion}

\section{Bridge graphs}

The bridge graph $\mathrm{B}_{1}=\mathrm{B}_{1}\left(\mathrm{G}_{1}, \mathrm{G}_{2}, . ., \mathrm{G}_{\mathrm{d}} ; \mathrm{v}_{1}, \mathrm{v}_{2}, \ldots, \mathrm{v}_{\mathrm{d}}\right)$ of a set of connected graphs $\left\{G_{i}: 1 \leq i \leq d\right\}$ is the graph obtained from $G_{1}, G_{2}, \ldots, G_{d}$ by connecting the vertices $\mathrm{v}_{i}$ and $\mathrm{v}_{\mathrm{i}+1}$ by an edge for all $i=1,2, . ., d$.

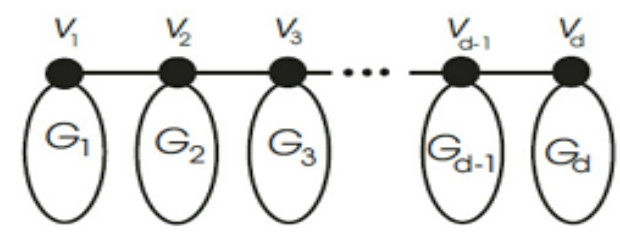

FIGURE 1 Bridge graph $\mathrm{B}_{1}$

The bridge graph $B_{2}=B_{2}\left(G_{1}, G_{2}, \ldots, G_{d} ; \quad v_{1}, w_{1}\right.$, $\left.v_{2}, W_{2}, \ldots, v_{d}, w_{d}\right)$ of a set of connected graphs $\left\{G_{i}: 1 \leq i \leq d\right\}$ is the graph obtained from $\mathrm{G}_{1}, \mathrm{G}_{2}, \ldots, \mathrm{G}_{\mathrm{d}}$ by connecting the vertices $\mathrm{w}_{\mathrm{i}}$ and $v_{i+1}$ by an edge for all $i=1,2, \ldots, d$.

Lemma-1: For $1 \leq i \leq d, d_{2}\left(u: B_{1}\right)=$ $\left\{\begin{array}{l}\gamma_{1}+\mu_{2}+1, \text { if } u=v_{1} \\ \gamma_{d}+\mu_{d-1}+1, \text { if } u=v_{d} \\ \gamma_{2}+\mu_{1}+\mu_{3}+1, \text { if } u=v_{2} \\ \gamma_{d-1}+\mu_{d}+\mu_{d-2}+1, \text { if } u=v_{d-1} \\ \gamma_{i}+\mu_{i-1}+\mu_{i+1}+2, \text { if } u=v_{i}, 3 \leq i \leq d-2 \\ d_{2}\left(u: G_{1}\right)+1, \text { if } u \in N\left(v_{1}: G_{1}\right) \\ d_{2}\left(u: G_{d}\right)+1, \text { if } u \in N\left(v_{d}: G_{d}\right) \\ d_{2}\left(u: G_{i}\right)+2, \text { if } u \in N\left(v_{i}: G_{i}\right), 2 \leq i \leq d-1 \\ d_{2}\left(u: G_{i}\right), \text { if } u \in V\left(G_{i}\right) \backslash N\left[v_{i}: G_{i}\right], 1 \leq i \leq d\end{array}\right.$ where $\gamma_{i}=d_{2}\left(v_{i}: G_{i}\right)$ and $\mu_{i}=\operatorname{deg}\left(v_{i}: G_{i}\right)$,

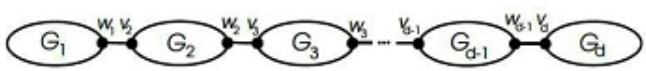

FIGURE 2 Bridge graph $\mathrm{B}_{2}$ 


\section{Theorem 1:}

$$
\begin{aligned}
& L F\left(B_{1}\right)=\sum_{i=1}^{d} L F\left(G_{i}\right)+2 \sum_{i=1}^{d} \mu_{i}^{3}-\mu_{1}^{3}-\mu_{d}^{3}+ \\
& 3 \sum_{i=1}^{d} L M_{1}\left(G_{i}\right)-3 \sum_{i=1}^{d} \sum_{u \in V\left(G_{i}\right) \backslash N\left[v_{i} G_{i}\right]} d_{2}\left(u: G_{i}\right)^{2}+3 \sum_{i=3}^{d-2} \gamma_{i}^{2} \\
& +3 \sum_{i=2}^{d-1} \sum_{u \in N\left(v_{i} G_{i}\right)} d_{2}\left(u: G_{i}\right)^{2}+3 \sum_{i=1}^{d-1}\left(\gamma_{i}^{2} \mu_{i+1}+\gamma_{i+1}^{2} \mu_{i}\right) \\
& +3 \sum_{i=1}^{d-1}\left(\gamma_{i} \mu_{i+1}^{2}+\gamma_{i+1} \mu_{i}^{2}\right)+\sum_{i=2}^{d-1}\left[1 2 \left(\mu_{i}^{2}+\gamma_{i} \mu_{i+1}\right.\right. \\
& \left.\left.+\gamma_{i} \mu_{i-1}\right)+6 \gamma_{i} \mu_{i-1} \mu_{i+1}+3 \mu_{i-1} \mu_{i+1}\left(\mu_{i-1}+\mu_{i+1}\right)\right] \\
& +12 \sum_{i=3}^{d-2} \gamma_{i}+32 \sum_{i=4}^{d-3} \mu_{i}+3 \sum_{i=1}^{d} \sum_{u \in N\left(v_{i} G_{i}\right)} d_{2}\left(u: G_{i}\right) \\
& +9 \sum_{i=2}^{d-1} \sum_{u \in N\left(v_{i}: G_{i}\right)} d_{2}\left(u: G_{i}\right)+3\left(\gamma_{1}+\gamma_{2}+\gamma_{d-1}+\gamma_{d}+\right. \\
& \left.\mu_{1}^{2}+\mu_{d}^{2}-\mu_{2}^{2}-\mu_{3}^{2}-\mu_{d-2}^{2}-\mu_{d-1}^{2}\right)+4\left(\mu_{1}+\mu_{d}\right) \\
& +6\left(\gamma_{1} \mu_{2}+\gamma_{d} \mu_{d-1}-\gamma_{2} \mu_{3}-\gamma_{2} \mu_{1}-\gamma_{d-1} \mu_{d-2}\right. \\
& \left.-\gamma_{d-1} \mu_{d}-\mu_{1} \mu_{3}-\mu_{d} \mu_{d-2}\right)+23\left(\mu_{2}+\mu_{3}+\mu_{d-2}\right. \\
& \left.+\mu_{d-1}\right)+8 d-28 .
\end{aligned}
$$

Proof: The proof of this result follows the definition of F-leap index and Lemma 1 through some algebraic operations.

\section{Chain graph}

Farkhanda Afzal et al. [3] discussed the weighted entropy of penta chains. Here we discussed the F-leap index of a more general chain graph.

The chain graph $\boldsymbol{C}=\boldsymbol{C}\left(G_{1}, G_{2}, \ldots, G_{d}\right.$ $\left.v_{1}, w_{1}, v_{2}, w_{2}, . ., v_{d}, w_{d}\right) \quad$ of $\quad\left\{G_{i}: 1 \leq i \leq d\right\} \quad$ with respect to the vertices $\left\{v_{i}, w_{i}: 1 \leq i \leq d\right\}$ is the graph obtained from the graphs $\left\{G_{i}: 1 \leq i \leq d\right\}$ by identifying the vertices $w_{i}$ and $v_{i-1}$ for all $1 \leq i \leq d-1$.

Lemma 2: Let $\left\{G_{i}: 1 \leq i \leq d\right\}$ be $C_{3}$-free connected graphs for $d \geq 5$ and let $\mathbf{C}$ be the chain graph formed using these graphs. Then the 2-degree of any arbitrary vertex $\mathbf{u}$ in $\mathbf{C}$ is given as follows: $d_{2}(u: \mathbf{C})=$

$$
\left\{\begin{array}{l}
d_{2}\left(u: G_{1}\right), \text { if } u \in V\left(G_{1}\right) \backslash N\left[w_{1}: G_{1}\right] \\
d_{2}\left(u: G_{1}\right)+\mu_{2}, \text { if } u \in N\left(w_{1}: G_{1}\right) \\
d_{2}\left(u: G_{d}\right), \text { if } u \in V\left(G_{d}\right) \backslash N\left[v_{d}: G_{d}\right] \\
d_{2}\left(u: G_{d}\right)+\lambda_{d-1}, \text { if } u \in N\left(v_{d}: G_{d}\right) \\
d_{2}\left(u: G_{i}\right), \text { if } \\
\begin{array}{l}
u \in V\left(G_{i}\right) \backslash\left\{N\left[w_{i}: G_{i}\right] \cup N\left[v_{i}: G_{i}\right], 2 \leq i \leq d-1\right. \\
d_{2}\left(u: G_{i}\right)+\mu_{i+1}, \text { if } u \in N\left(w_{i}: G_{i}\right) \backslash N\left(v_{i}: G_{i}\right),
\end{array} \\
\quad 2 \leq i \leq d-1 \\
d_{2}\left(u: G_{i}\right)+\lambda_{i-1}, \text { if } u \in N\left(v_{i}: G_{i}\right) \backslash N\left(w_{i}: G_{i}\right), \\
\quad 2 \leq i \leq d-1 \\
d_{2}\left(u: G_{i}\right)+\lambda_{i-1}+\mu_{i+1}, \text { if } \\
u \in N\left(v_{i}: G_{i}\right) \cap N\left(w_{i}: G_{i}\right), 2 \leq i \leq d-1 \\
\delta_{i}+\gamma_{i+1}, \text { if } u=w_{i}=v_{i+1}, 1 \leq i \leq d-1
\end{array}\right.
$$

where $\gamma_{i}=d_{2}\left(v_{i}: G_{i}\right), \delta_{i}=d_{2}\left(w_{i}: G_{i}\right)$, $\mu_{i}=\operatorname{deg}\left(v_{i}: G_{i}\right)$ and $\lambda_{i}=\operatorname{deg}\left(w_{i}: G_{i}\right)$ for all $1 \leq i \leq d$.

Theorem 2: $\operatorname{LF}(\mathrm{C})=$

$\sum_{i=1}^{d} L F\left(G_{i}\right)+3 \mu_{2} \sum_{u \in N\left(w_{1}: G_{1}\right)} d_{2}\left(u: G_{1}\right)\left[d_{2}\left(u: G_{1}\right)\right.$

$\left.+\mu_{1}\right]+3 \lambda_{d-1} \sum_{u \in N\left(v_{d}: G d\right)} d_{2}\left(u: G_{d}\right)\left[d_{2}\left(u: G_{d}\right)+\lambda_{d-1}\right]$

$+\sum_{i=2}^{d-1} \sum_{u \in N\left(w_{i}: G_{i}\right) \backslash N\left(v_{i}: G_{i}\right)}\left(3 d_{2}\left(u: G_{i}\right) \mu_{i+1}\left[d_{2}\left(u: G_{i}\right)+\mu_{i+1}\right]\right.$ $\left.\mu_{i+1}^{3}\right)+\sum_{i=2}^{d-1} \sum_{u \in N\left(v_{i} G_{i}\right) N\left(w_{i}: G_{i}\right)}\left(3 d_{2}\left(u: G_{i}\right) \lambda_{i-1}\left[d_{2}\left(u: G_{i}\right)+\lambda_{i-1}\right]+\lambda_{i-1}^{3}\right)$ $+3 \sum_{i=2}^{d-1} \sum_{u \in N\left(v_{i}: G_{i}\right) \cap N\left(w_{i}: G_{i}\right)}\left(d_{2}\left(u: G_{i}\right) \lambda_{i-1}\left[d_{2}\left(u: G_{i}\right)+\lambda_{i-1}\right]\right.$ $+d_{2}\left(u: G_{i}\right) \mu_{i+1}\left[d_{2}\left(u: G_{i}\right)+\mu_{i+1}\right]+\lambda_{i-1}^{3}+\mu_{i+1}^{3}$

$\left.+6 \lambda_{i-1} \mu_{i+1} d_{2}\left(u: G_{i}\right)\right)+3 \sum_{i=2}^{d-1} \lambda_{i-1} \mu_{i+1}\left[\lambda_{i-1}+\mu_{i+1}\right]$ $+\sum_{i=1}^{d-1} \delta_{i}^{3}+3 \delta_{i} \gamma_{i+1}\left[\delta_{i}+\gamma_{i+1}\right]+\lambda_{1} \mu_{2}^{3}+\lambda_{d-1}^{3} \lambda_{d}$.

Proof: By definition of F-leap index and applying Lemma-2 through algebraic calculations we get the required result.

Special classes of bridge graph $B_{1}$

Let $\mathrm{B}_{1}(\mathrm{P})$ denote the bridge graph $B\left(P_{n_{1}}, P_{n_{2}}, \ldots, P_{n_{k}} ; v_{1}, v_{2}, \ldots, v_{k}\right)$ formed using 
paths $P_{n_{i}}$ on $\left\{n_{i}: 1 \leq i \leq k\right\}$ vertices by identifying a vertex $v_{i}$ in each such path and joining $v_{i}$ with $v_{i+1}$.

Theorem 3: $L F\left(B_{1}(P)\right)=8 \sum_{i=1}^{k} n_{i}+103 k-318$

Proof: We first observe the vertex partition of $B_{1}(P)$.

TABLE 1 Vertex partition of $\mathrm{B}_{1}(\mathrm{P})$

\begin{tabular}{cc}
\hline 2-degree & \#vertices \\
\hline 1 & $2 \mathrm{k}$ \\
2 & $\sum_{i=1}^{k}\left(n_{i}-3\right)$ \\
3 & 2 \\
4 & 2 \\
5 & $\mathrm{k}-4$ \\
\hline
\end{tabular}

By the definition of F-leap index we have $L F\left(B_{1}(P)\right)=\sum_{v \in V\left(B_{1}(P)\right)} d_{2}(v)^{3}$.

By applying the 2-degrees given in Table 1, $L F\left(B_{1}(P)\right)=8 \sum_{i=1}^{k} n_{i}+103 k-318$.

Corollary: If $n_{1}=n_{2}=\ldots=n_{k}=r$, then we denote the bridge graph in this case by $B_{r, k}$.Now $L F\left(B_{r, k}\right)=8 r k+103 k-318$.

Let $\mathrm{B}_{1}(\mathrm{~K})$ denote the bridge graph $B\left(K_{n_{1}}, K_{n_{2}}, \ldots, K_{n_{k}} ; v_{1}, v_{2}, \ldots, v_{k}\right) \quad$ over the complete graphs $K_{n_{i}} ; 1 \leq i \leq k$.

\section{Theorem-4:}

$L F\left(B_{1}(K)\right)=\sum_{i=3}^{k-2}\left(n_{i-1}+n_{i+1}\right)^{3}+8 \sum_{i=2}^{k-1} n_{i}+\left(n_{2}^{3}+n_{k-1}^{3}\right)$ $+\left(n_{1}+n_{3}-1\right)^{3}+\left(n_{k-2}+n_{k}-1\right)^{3}+\left(n_{1}+n_{k}-8 k+14\right)$.

Proof: It is easy to note that there are $\left(n_{1}+n_{k}-2\right)$ vertices of 2 -degree one and $\sum_{i=2}^{k-1}\left(n_{i}-1\right)$ vertices of 2 -degree two in $B_{1}(K)$.Further we obtain the 2-degree of the vertices $v_{i}$ as follows:
TABLE 2 2-degree of vertices in $\mathrm{B}_{1}(\mathrm{~K})$

\begin{tabular}{cc}
\hline Vertex v & $d_{2}(v)$ \\
\hline$v_{1}$ & $n_{2}$ \\
$v_{2}$ & $n_{1+} n_{3}-1$ \\
$v_{i}$ & $n_{i-1}+n_{i+1}, 3 \leq i \leq k-2$ \\
$v_{k-1}$ & $n_{k-2}+n_{k}-1$ \\
$v_{k}$ & $n_{k-1}$
\end{tabular}

Now, based on the definition of F-leap index, we have following results:

Corollary: If $n_{1}=n_{2}=\ldots=n_{k}=n$, then $L F\left(B_{1}(K)\right)=8(n-1) k+(8 k-14) n^{3}-24 n^{2}$ $-2 n+12$.

\section{Applications}

(i) The square comb lattice $C_{q}(N)$ having open ends and $N=n^{2}$ vertices can be viewed as a bridge graph $B_{n, n}$.

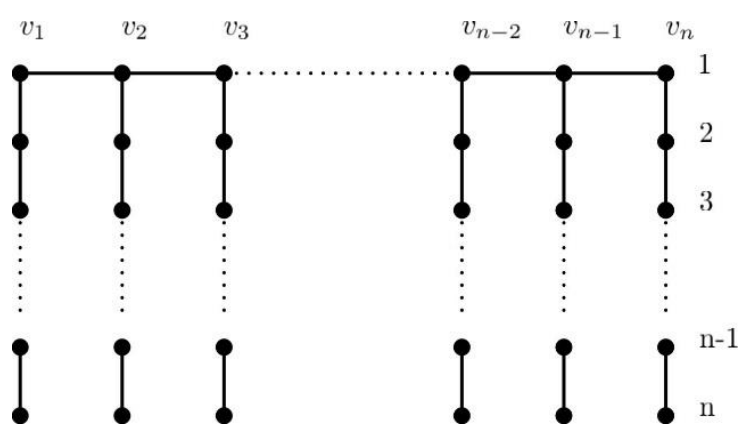

FIGURE 3 Square comb lattice

Therefore by corollary of Theorem-3, we get $L F\left(C_{q}(N)\right)=8 n^{2}+103 n-318$.

(ii) Consider the van Hove comb lattice graph $\mathrm{CvH}(\mathrm{N})$ with open ends. This graph can be represented as the bridge graph $B_{1}\left(P_{1}, P_{2}, P_{3}, \ldots, P_{n-1}, P_{n}, P_{n-1,} P_{n-2}, \ldots, P_{2}, P_{1} ; v_{1}\right.$, $\left.v_{2}, \ldots, v_{n}, u_{n-1}, \ldots, u_{1}\right)$.

Here we observe the following table. So, applying Theorem-1, we get $L F(C v H(N))=8 n^{2}+244 n-634$.

(iii) Two vertices $v$ and $w$ of a cycle $C$ are said to be in 
(a) ortho-position, if they are adjacent in C

(b) meta-position, if they are distance two in $\mathrm{C}$

(c) para-position, if they are distances three in C.

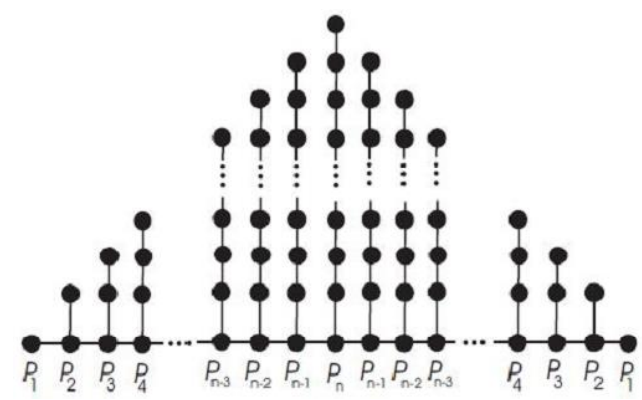

FIGURE 4 Van Hove comb lattice $\mathrm{CvH}(\mathrm{N})$

TABLE 3 Vertex partition in $\mathrm{CvH}(\mathrm{N})$

\begin{tabular}{cc}
\hline 2-degree & \#vertices \\
\hline 5 & $2 n-5$ \\
3 & $2 n-7$ \\
2 & $2 \sum_{i=4}^{n-1} i-7 n+36$ \\
& $4 n-12$ \\
\hline
\end{tabular}

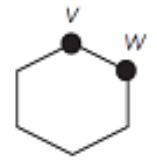

Ortho-position

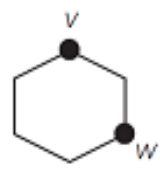

Meta-position

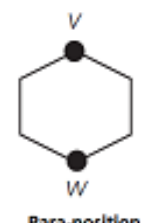

Para-position
FIGURE 5 Ortho, meta, para positions in a cycle.

Theorem 5. The F-leap index of bridges over k cycles of different lengths $n_{i}>4$ in ortho position is

$$
L F\left(B_{1}(C)\right)=8 \sum_{i=1}^{k} n_{i}+616 k-1260 .
$$

We decide this computation from Table 4.

TABLE 4 Vertex partition in $\mathrm{B}_{1}(\mathrm{C})$.

\begin{tabular}{cc}
\hline 2-degree & \#vertices \\
\hline 2 & $\sum_{i=1}^{k}\left(n_{i}-3\right)$ \\
3 & 4 \\
4 & $2 k-4$ \\
5 & 2 \\
7 & 2 \\
8 & $k-4$ \\
\hline
\end{tabular}

Corollary: In particular, if $n_{i}=n, 1 \leq i \leq k$, then $L F\left(B_{1}(C)\right)=8 n k+616 k-1260$.

Theorem 6: The F-leap index of bridge graph over $\mathrm{k}$ cycles of different lengths $n_{i} \geq 5$ in meta-position is $L F\left(B_{2}(C)\right)=8 \sum_{i=1}^{k} n_{i}+188 k-188$.

Proof: The proof is straight forward using the following vertex partition of $B_{2}(C)$.

Table 5 Vertex partition of $B_{2}(C)$

\begin{tabular}{cc}
\hline 2-degree & \#vertices \\
\hline 2 & $\sum_{i=1}^{k} n_{i}-6 k+6$ \\
3 & $4 k-4$ \\
4 & $2 k-2$ \\
\hline
\end{tabular}

Theorem 7: The F-leap index of bridge graph over $\mathrm{k}$ cycles of different lengths $n_{i} \geq 9$ in para-position is

$L F\left(B_{2}(C)\right)=8 \sum_{i=1}^{k} n_{i}+188 k-188$.

Proof: The proof of this result is just the same as that of Theorem 6 .

The polyphenyl chain of $h$ hexagons is said to be an ortho- $\left(\mathrm{O}_{h}\right)$, meta- $\left(\mathrm{M}_{h}\right)$ and para- $\left(\mathrm{L}_{\mathrm{h}}\right)$, if all its internal hexagons are ortho-hexagons, meta-hexagons and Para-hexagons, respectively.
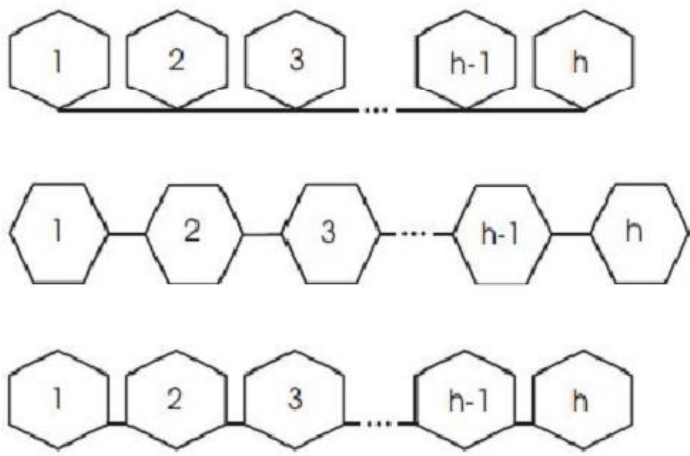

FIGURE 6 Polyphenyl chains in ortho, meta, para positions 
The polyphenyl chain $\mathrm{O}_{\mathrm{h}}$ is represented as the bridge graph $B_{1}=B_{1}\left(C_{6}, \ldots, C_{6} ; v, v, . . v(\mathrm{~h}\right.$ times $\left.)\right)$. Therefore by corollary of Theorem 5, $L F\left(O_{k}\right)=664 h-1260$.

Similarly, the polyphenyl chains Mh and Lh are represented as the bridge graph $B_{2}=B_{2}\left(C_{6}, C_{6}, \ldots, C_{6}, v, v, \ldots v\right.$ (h times)). Therefore by corollary of Theorem 6 (or Theorem 7) we get $L F\left(M_{h}\right)=L F\left(L_{h}\right)=236 h-188$.

The spiro chain $\operatorname{SP}\left(C_{n}, d\right)$ is the chain graph $\boldsymbol{C}=\boldsymbol{C}\left(C_{n}, C_{n}, \ldots, C_{n} ; v, w v, w, \ldots, v, w\right)$. The spiro chains of $C_{4}$ and $C_{6}$ are given by Figure

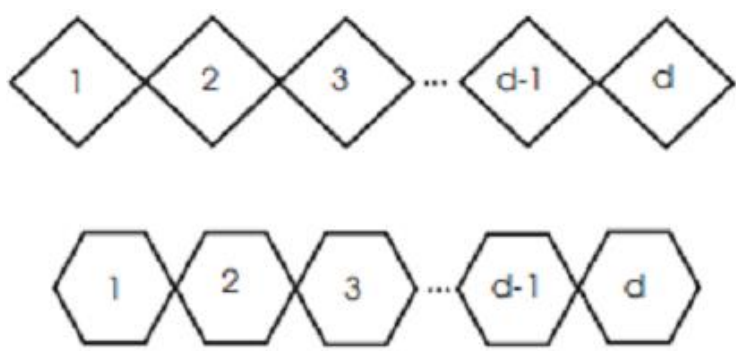

FIGURE 7 Spiro chains $\mathrm{SP}\left(\mathrm{C}_{4}, \mathrm{~d}\right)$ and $\mathrm{SP}\left(\mathrm{C}_{6}, \mathrm{~d}\right)$

Clearly, $\quad \operatorname{LF}\left(\operatorname{SP}\left(C_{4}, d\right)\right)=136 d-154$ and $\operatorname{LF}\left(S P\left(C_{6}, d\right)\right)=264 d-160$.

\section{Conclusion}

In this work, F-leap index of bridges and chain graphs were analyzed and the topological invariant was computed for some special types of bridge graphs. Several applications of these generalized graph structures were highlighted, as well. One may try to correlate these results with chemical compounds which have similar structure such as bridge graphs and chain graphs to obtain fruitful results before conducting the experiments.

\section{Acknowledgments}

The authors are thankful to Dr. Mehdi Alaeiyan and Mohammad Reza Farahani of Department of Mathematics, Iran University of Science and Technology (IUST) for their precious support and suggestions.

\section{Orcid:}

Mohanad Ali Mohammed:

https://orcid.org/0000-0002-7507-1212

Janet Robert:

https://orcid.org/0000-0002-2340-9840

Natarajan Chidambaram:

https://orcid.org/0000-0002-1001-3556

Narasimhan Devadoss:

https://orcid.org/0000-0001-9375-3754

\section{References}

[1] M. Azari, A. Iranmanesh, I. Gutman, MATCH Commun. Math. Comput. Chem., 2013, 70, 921938.

[2] J. Devillers, A.T. Balaban, Eds., Gordon and Breach, 1999, Amstersam, the Netherlands.

[3] F. Afzal, M.A. Razaq, D. Afzal, S. Hameed, Eurasian Chem. Commun., 2020, 2(6), 652662.

[4] B. Furtula, I. Gutman, J. Math. Chem., 2015, 53, 1184-1190.

[5] I. Gutman, N. Trinajstić, Chem. Phys. Lett., 1972, 17, 535-538.

[6] V.R. Kulli, Int. J. Math. Archive, 2018, 9, 4149.

[7] A.M. Naji, N.D. Soner, I. Gutman, Commun. Comb. Optim, 2017, 2, 99-117.

[8] N. De, Mal. J. Fund. Appl. Sci. 2016, 4, 109113.

[9] Z. Shao, I. Gutman, Z. Li, S. Wang, Pu Wu, Commun. Comb. Optim, 2018, 3, 179-194.

[10] X. Zhang, H.Q. Jiang, J.B. Liu, Z.H. Shao, Molecules, 2018, 23, 1731-. DOI: 10.3390/molecules23071731

[11] M. Alaeiyan, C. Natarajan, G. Sathiamoorthy, M.R. Farahani, Eurasian Chem. Communm., 2020, 2, 646-651.

[12] H. Yang, X. Zhang, J. Dis. Math. Sci. Crypt. 2018, 21, 1495-1507.

[13] M. Imran, S.A. Bokhary, S. Manzoor, M.K. Siddiqui. Eurasian Chem. Communm., 2020, 2, 680-687.

[14] Z. Ahmad, M. Naseem, M.K. Jamil, Sh. Wang, M.F. Nadeem, Eurasian Chem. Commun., 2020, 2, 712-721. 
[15] Z. Ahmad, M. Naseem, M.K. Jamil, M.K. Siddiqui, M.F. Nadeem. Eurasian Chem. Commun., 2020, 2, 663-671

[16] M. Cancan, S. Ediz, M.R. Farahani, Eurasian Chem. Commun., 2020, 2, 641-645.

[17] A.Q. Baig, M. Naeem, W. Gao, J.B. Liu, Eurasian Chem. Commun., 2020, 2, 634-640.
How to cite this article: Mohanad Ali Mohammed, Raad Sehen Haoer, Janet Robert, Natarajan Chidambaram*, Narasimhan Devadoss. F-leap index of some special classes of bridge and chain graphs. Eurasian Chemical Communications, 2020, 2(7), 827-833. Link:

http://www.echemcom.com/article_107 560.html

Copyright (C) 2020 by SPC (Sami Publishing Company)+ is an open access article distributed under the Creative Commons Attribution License, which permits unrestricted use, distribution, and reproduction in any medium, provided the original work is properly cited. 